\title{
SLAC-FUB-3734
}

\section{A RESEARCH PROGRAM WTTH NO "MEASUREMENT PROBLEM"}

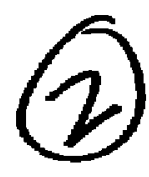

\author{
H. PIERrE Noyes \\ Stanford Linear Acederator Center \\ Stanford Univeruity, Stanjord, California, 94B05 \\ and \\ Cerugtorfer GeFWert \\ Aeademy of Finland, Helsinki $\quad-\% 94: 4=$ \\ and \\ MICaAEL J.MaNTHEY \\ Computer Seienee Department, Univ, of New Mexico
}

Submitted to the Proc. New Yark Acadamy of Sciences

"Now Techniques and Ideas in Quantum Mensurement Theory"

New York Cily, 21-24 January، 1986

- Wort mpported by the Depawtinent of Energy, controct DE - ACO3 - 76SF00515. 


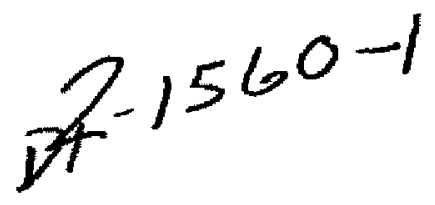

\section{CONF-860147--1}

The "raeamurement problem" of contemporary physica to met by recognizine that the phyzicist participates when conatrueting and (wheal applying the theory consisting of the formulated formal and measurament exiterie (the expressions and rakes) providing the neceseary conditiont which allow him to cornpute and mearure fucts, yet retains objectivity by requiring that these criterito, fules and facts be in corroborative equilibrium. We constret the purticulate states of quantum physica by a recursive program which ipcorporates the non-determinitm born of communication between asynchronoul processen orer a ubared memory. Their quantum numbers and coupling constents arise from the construction tis the unique f-ltuel combinatorial hierarehy. The constraction doftnou indivialble quantum events with the requisite supreluminal correlatlops, yet does not allow supraluminal communication. Meacurement crituris ineorporate $c, h$ and $m_{p}$ or (not "and") G. The resulting thtory is dacreto throughont, contuins do Indinttes, and, as far as we have developed it, is in agrement with quartum mechanleal and cosmological fact.

\section{DISCLAMER}

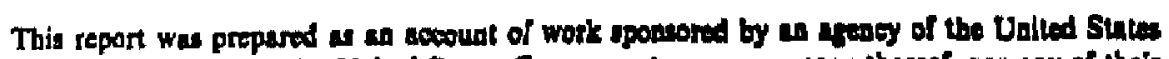

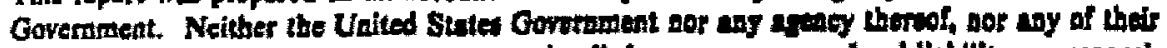

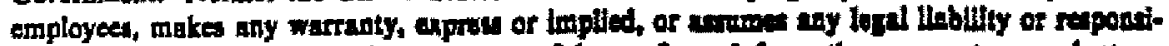

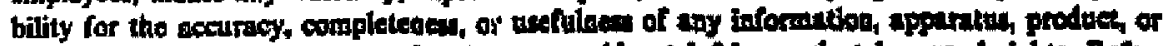

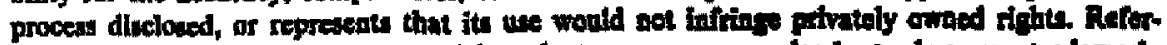

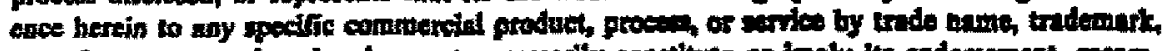

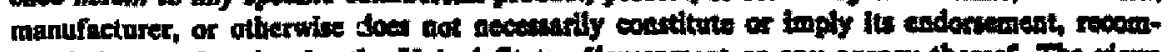
mendation, or favortog by the United States Goverameat of any ayency thereof. The view

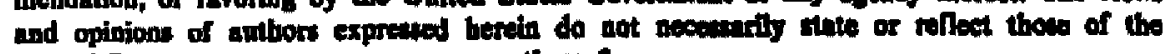
United Stutes Goversmeat of any weptoy thenof. 


\section{INTRODUCTION}

Quantum mechanics has to be adjoined to a "measurement theory" that has never been formulated in s satirfactory way; it is in the words of Wheeler ${ }^{|l|}$ a law without law. For ws "measurement" is part of any research prograng in physics; if we coustruct phynie as a jeseanch progran the "measurement problem" cannot be given a separate loeas. We $\mathrm{W}^{|3|}$ first formulate what we mean by a participatory sesearch program that opecifies the criteria and the steps which can allow us to conclude that the program is complete. We present this schema in fig. 1 . The inglied philonophical position bas been discussed by one of us (CG) elsewhere ${ }^{|2|}$ - Athough participation is involved in the creation of the program in virtue of the meaning-conferring acts of judgment entailed, the end result is objective in that, if suecessful, the program provides the same explanation of meaning for any participant when applied.

The technique we use to thow the objectivity is to code the program and bence insure that it is computable. The program uses arbitrary numbers, in McGoveran's sense, generated by the non-determinism born of communication between arynchronous processes over a shared memory. . "The basic entities in the program are ordered atrings of the symbols " 0,1 " generated either by adding one arbitrary bit to each extant string or by discriminating between strings and adjoining a novel result to the bit string universe. The act of concatenating each axtant string with an arbitrary bit is our representation of a "quantum event", changing the entire bit string universe whenever discrimination between extant strings fals to produce demonstrable novelty. Clearly such events are non-local, which is currently an experinentally implied requirement for quantum events. The problem is zather to show that in the articulation of the theory they do not allow supralominal signalling.

- D.McGoveran wes "arbitray" to maan "not due to any finite, locally apecifiable algorithm"; rince compater hardware is Enite and atlempts to be locally deterninistic, he would replace Marhay'a term "non-determinimen" by the term "multi-determiniom" (privale communiontion]. 
The means used to connect the bit atring universe to the practice of particle physice is to essume that

any elementary event, under circumstances which it ia the task of the czperimental physicist to investigate, can lead to the firing of a counter.

We call this the "counter paradigm". It allows us to connect the "quantum eventg" which occur in our computer program with laboratory counter firings in such a way as to provide our theory with both predictive power and corriglbility. We identify the three or Cour bit Etrings deffning any quantum event as the basis states needed to construct a finite particle number relativistic (i.c. constrained by the "limiting velocity for signals") quantum scattering theory, including the conserved quantum numbers encountered in the "standard model" of quarka and leptons, and to make a start on computing the scale constants of modern physics.

\section{CONSTRUCTING A BIT STFUNG UNIVERSE}

The basic entities in the theory are ordered strings of the symbols " 0 ", "1" [labeled below by $a, b, \ldots]$ defined by $S^{a}\left(N_{V}\right)=\left(\ldots, b_{n}^{a}, \ldots\right) N_{v}$, where $b_{n}^{a} \in 0,1$ and $n \in\left[1,2, \ldots, N_{U}\right]$. The strings combine by "XOR" : $S^{a} \oplus S^{b} \equiv\left(\ldots, b_{n}^{a}+2 b_{n}^{b} \ldots\right) N_{U}$ (for 0,1 bits) ar by $S^{a} \oplus S^{b} \equiv\left(\ldots,\left(b_{n}^{a}-b_{n}^{b}\right)^{2}, \ldots\right)_{N_{t}}$ (for 0,1 integers). This fruitfu] ambiguity allows us to refer to either operation as diseriminalion. The null string is called $D_{N} \equiv(0,0, \ldots, D)_{N}, S^{a} \oplus S^{a}=0_{N i}$ the anti-null atring is symbolized by $1_{N} \equiv(1,1, \ldots, 1)_{N}$, allowirg us to define the "bar operation"

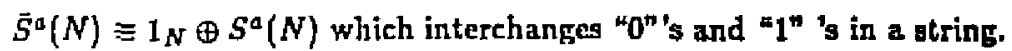

We generate the strings according to the How chart, fig. 2 . The program is initiated by the arbitrary choice of two distinct bits; $R:=0$ or $1, R=1 \oplus R$, Entering at $P I C K$, we take $S_{1}:=P I C K ; S_{2}:=P J C K ; S_{12}:=S_{1} \oplus S_{2}$. If $S_{12}=0_{N_{v}}$, we recurse to picking $S_{2}$ until we pass this test. We then ask if $S_{12}$ is already in the universe. If it is not we adjoin it, $U:=U U S_{1 z}, S U:=s U+1$, and return to PICK. If $S_{12}$ is already in the universe, we go to our third, and last, arbitrary operation called $T I C K$. This simply adjoins a bit (via $R$ ), arbitrarily chosen for each string, to the growing end of each string, $U:=U \| R, N:=N+1$, 
and returns us to PICK; here "fl" denotes atring concatenation. TICK results either from a s-evert which guarantees that at that $N_{U}$ the universe contains three strings constrained by $S^{\star} \oplus S^{b} \oplus S^{c}=0_{N_{I I}}$ or a f-event constrained by $S^{d} \oplus S^{b} \oplus S^{c} \oplus S^{d}=0_{N_{v}}$ That these are the only ways events happen in the bit string universe is demonstrated in fig. 3 .

Given two distinct (linearly independent or h,i.) non-nut!' zrings $a, b$, the set $\{a, b, a \oplus b\}$ closes under discrimination. Observing that the singleton sets $\{a\}$, $\{b\}$ are closed, we see that two l.j. strings generate three discriminately closed subsets (DCsS's). Gives a third I.i. string $c$, we can generate $\{c\},\{t, c, b \oplus c\}$, $\{c, a, c \oplus a\}$, and $\{a, b, c, a \oplus b, b \oplus c, c \oplus a, a \oplus b \oplus c\}$ as well. In fact, given $j$ li. strings, we can generate $2^{j}-1$ DCsS's because this is the number of ways we can choose $j$ distinct things one, two,... up to $j$ at a time. This allows us to construct the combinatorial hierarchy ${ }^{i s !}$ by generating the sequence $\left(2 \Rightarrow 2^{2}-1=3\right),(3 \Rightarrow$ $\left.2^{3}-1=i\right),\left(7 \Rightarrow 2^{\tau}-1=127\right),\left(127 \Rightarrow 2^{12 \tau}-1 \simeq 1.7 \times 10^{38}\right)$ mapped by the sequence $\left(2 \Rightarrow 2^{2}=4\right),\left(4 \Rightarrow 4^{2}:=16\right),\left(16 \Rightarrow 16^{2}=256\right),\left(256 \Rightarrow 256^{2}\right)$. The process terminates because there are only $256^{2}=65,536=6.5536 \times 10^{4}$ l.i. matrices available to map the fourth level, which are many too few to map the $2^{127}-1=1.7016 \ldots \times 10^{38}$ DCsS's of that level. This (unique) hierarchy is exhibited in Table 1 . The closure of the hierarchy allows us to divide the strings generated by the program into a finite initial segment (called th label) and a growing remainder. The labels close in some representation of the 4-lovel combinatorial hierarchy with oxactly $2^{127}+136$ strings of fxed length, which are then used to label address ensembles, as is discussed in more detail in Ref. 2 and elsewhere.

Each event regults in a TICK, which increases the complexity of the universe in an irreversible way. Our theory has an ordering parameter $\left(N_{U}\right)$ which is conceptually closer to the "time" in generil relativistic cosmologies than to the "reversible" time of special relativity. The arbitrary elements in the algorithm that generates events preclude unique "retrodiction", while the finite complexity parameters $\left(S U, N_{U}\right)$ prevent a combinatorial explosion in statistical retrodiction. 
In this sense we have a fired - though only partially retrodictable - post and a necessarily unknown future of finite, but arbitrarily increasing, complexity. Only structural characteristics of the system, rather than the bit strings used in computer sinulations of pieces of our theory, are available for epistemological correlations with experience.

\section{SCATTERING THEORY}

Now that we have eatablighed the formal elemants of the theory and the rules that allow us to compute formal facts, we must establish measurement criteria. This is done by relating the bit strings to the basis states of a relativistic, unitary and "crosaing symmetric" quantum particle scattering theory, and deriving the "propagator" of that theory which connects events as some gystem within the universe evolves. The labels are used to define quantum numbers - symmetric botween "particles" and "antiparticles" - that are conserved in connected events. The labeled address strings are interpreted as the velocities associated with these quantum numbers; by nppropriate definition they are measured in units of the limiting velocity " $c$ ". Since quantum scattering theory asgociates quantum numbers with discrete conserved masses, and 3-momenta conserved in evolying eystems, we also use the labeled addiress strings to define velocitios (in urits of the limiting velocity) which when multiplied by the appropriate discrete masses coneerve 3 -momentum in the discrete " $3+1$ space" that our events allow us to construct. Since the labels close these quantum numbers and masses $m_{w}$ (which it will become the task of the theory to compute self-consistently) retain an invariant significance no matter how long the program runs, or bow long and large the address striag ensembles become.

The scattering theary on which we rely ${ }^{|s|}$ starts from three distinguishable particles and a linear, unitary quantum dynamies based on relativistic threeparticle Faddeev equations (which can be viewed as the summation of quantum cvents with approprjate statistical weights). The basic entities for "Yulawa coutpling" are a particle, an antiparticle (number of particies minus number of entiparticle conserved), and a quastum (with zero particle quantum number) to 
which this pair can conlesce, or which can disassociate into the pair; a quanturn can be ninitted or absorbed by a particle (or anti-particle) without changing the particle quantum number. Particles and quanta may carry other conserved quasltum numbers allowing a definition of "anti-guanta", but there must always be one quantum state which carries only null quantum numbers. The "quantum" associated with that state is indistinguishable from its "anti-quantum".

We symbolize any string by $S^{w}=\left[L^{w}\left(N_{L}\right), A^{w}(N)\right]$. Our basic quantum number acheme for three linearly independent strings of bit length $A$ is given in fig. 4 , which meets the requirements set above. For any address string $A^{\mathrm{w}}(N)$, the parameter $k_{w}=\Sigma_{n=1}^{N} b_{n}^{\mathrm{w}}$ allows us to define a signed rational fraction $\beta_{v}$ for each address string by taking $2 k_{w}=N\left(1+\beta_{v}\right)$; clearly $\beta_{w} \in$ $\left[-1, \frac{-(N-1)}{N}, \ldots . ., \frac{(N-1)}{N},+1 \mid\right.$. Thus a 3 -event initiates a state $\mid N ; k_{\mathrm{a}}, k_{\mathrm{b}}, k_{\mathrm{c}}>$ defined by four integers (referring to the address string, and at least two quantum numbers each for $a, b$, and $c$ as discussed above) which specify three scalar rational fractions; these we interpret as velocities in units of the limiting velocity c.

Since the basic discriminations also define the strings $A^{a b}=A^{a} \oplus A^{b}=A^{c}$ ( $a, b, c$ cyclic), and hence $\beta_{a b}=\beta_{c}$ we conclude that each pair has the same velocily as the third, or spectator, bystem. The three velocjties, three pair velocities, and three masses provide 9 of the 12 degrees of freedom of the three 4-vectors in a conventional description, while the remaining three cannot be specified without specific context because our construction has geometrical isotropy. We note that the "bar" operation $\bar{S}=\mathbf{1}_{N_{\mathrm{U}}} \oplus S$ reverses the sign of all velocities and all quantum numbers at the same time. In contrast, if we reverse only the velocities, the helicities do not reverse, showing that they are "pseudo-vectors". Our basis states have the characteristics needed for "crossing symmetry" and "CPT iлvariasce".

To obtain the statistical connection between events, we start from our counter paradigm, and note that because of the macroscopic size of laboratory counters, there will always be some uncertainty $\Delta \beta$ in measured velocities, reflected in our 
integera $k_{\mathrm{a}}$ by $\Delta k=\frac{1}{2} N \Delta \beta$. Thus, if we start with some specified spreaci of events corresponding to laboratory boundary conditions, and tick away, the fraction of connected everts we need consider diminishes in the manner illustrated in fig. 5 - Since the "off shell propagator" of quantum scattering theory refers to the probability that two states which do not conserve energy will be connected we claim that we could, given more space, conclude from this calculation that the propagator is proportional to $\frac{1}{\left(E-E^{4} \mp 0^{+}\right]}$.

Now that we have masees and the limiting velocity, and we know that from the hierarchy construction that the simplest unit of muss to use will be either the proton or the Planck mass, the only remaining dimensional constant to assign is the unit of axtion, or angular momentum. In previous treatments we have used the digital structure of the addregs strings and velocities to describe a drunkard ${ }^{t} s$ walk between events weighted by $\frac{1}{2}(1+\beta)$ with step length $h c / E$, which implies a coherence length $h / p$ and hence the usual relativistic Debroglie phase and group velocities. Mecent work on discrete topology by McGoveran ${ }^{(0)}$ makes it likely that the digital structure also implies the usual relation $\ell_{x} \ell_{y}=i \ell_{x}$ resulting from the "torsion" inherent in defining "distance" in a finite, digital space. Selfconsistent definition of $h, h$ and $\pi$ along this route is a formal criterion we hope to meet in the near future.

\section{THE STANDARD MODEL}

We interpret one dichotomous quantun. number for each nf the four levels as helicity. Since Level 1 has only two independent states, and these are coupled by the "bar" operation to the sign of the velocities which they label, we interpret these two basis states as chiral (two component) neutrinos. The next two quantum numbers (Level 2) allow for particle-antiparticle (or "chargen) discrimination with helicity $\pm \frac{1}{2}$ coupled to two \pm 1 helicity states and the degenerate $\left(0_{1}, 1_{4}\right)$ zero helicity atate. We take these to be charged leptons coupled to a massloss "spin $I^{n}$ quantum, and the assaciated "coulomb" interaction. If we were constructing a "field theory" this would restaict us to the "physical" or "couloinb" gauge. In a finite particle number theory with exact unitarity this is not a restriction but 
a coneeptual necestity.

For Level 3 we concatenate a string of length 4 (interpreted as defining particle and helicity states $q_{1}, q_{2}$ ) with a string defining the color octet. One way of getling the $\mathrm{SU}_{3}$ octet from our strings is given in Table 2 (or implied in Figure 4.). For color we could take red $=(0001)$, anti-red $=(1110)$; yellow $=(0010)$, anti-yellow $=$ (1101); blue $=(1100)$, anti-blue $=(0011)$. Then three colors or three anticolors give the color singlet (1111), as do the appropriate combinations of color and anti-color. The three besil strings so constructed give us a colored quark and the usocisted gluons. Since $a \oplus a \oplus a \equiv a$, three colored quarks (or anti-quarks) add to give a color singlet and yield the spin and helicity states of a nucleon (anti-nucleon). Doubling the first four bits gives us a second Gavor of quark, and a acond nucleon when we form a color singlet using two of the first type and one of the second. Details will be presented elsewhere. Speculatively, since the scattering theory employed allows three states of the same mass to combine to alngle state of thet mass, we can take both the quark and the nucleon mass to be the sarac; this would mean that quark structure would only appear at the 3 Gev level, which is desirable if nuclear physics is to continue to use mesons and rueleons as a frst approximation. Level four gives us a combinatorial explosion of higher generations with the aame structure, but only weakly coupled because of the Jarge number of combinatorial possibilities.

The Enal step at this stage in the development of our theory is to set the mass ratio scale by invoking the Parker-Rhodes calculation ${ }^{|7|}$. As we have argued sevaral times, our interpretation of quantum numbers and construction of $3+1$ "вpaca" allows us to take this over intect, and claim that $m_{p} / m_{c}=$ $\frac{1+7 x}{\langle x(1-y)><1 / y} ; 0 \leq x \leq 1 ; 0 \leq(1 / y) \leq 1$ where $x$ is the charge in unita of $e^{2}=n c / 137$ and $y$ is the radial distance from the center of symmetry limited from below by the minimal radial distance for a system at rest, $h / 2 m_{p} c$. The statistical calculation is straightforward, and for three degcees of freedorn gives $<x$ (1 x) $>=(3 / 14)\left[1+(2 / 7)+(2 / 7)^{2}\right]$ and $<1 / y>=4 / 5$. Hence $m_{r} / m_{c}$ is predicted to be 1836.151497 ... in comparison with the accepted value of $1836.1515 \pm 0.0005$. 
Although this result has been published and presented many times, we know of no published challenge to the calculation.

\section{CONCLUSTONS}

As we have said before|2|, "The idea of a theory as a theory of conotruction is valid independent of the "information conteat" of the theory. In order for a regearch program to succeed, it must create complete understanding in the way we have developed the theory. Whatever "machinery" in formulated an a theory of constructions, the participator idea implicit in the theory structure is necessary in order to understand.

"In this paper we have proved that by starting from bit stringe generated by program universe and labeled by the $2^{137}+136$ slaings provided by any representation of the four-level combinatorial hierarchy one gets an 5-matrix theory with the usua] $C, P, T$ properties, $C P T$ and crossing invariance, manifest covarlance and a candidate to replace quantum field theory by an $N$-particle scattering theory which will not be in conflict with practice for some sutficlently large finite $N$. Arbitsary ("random") choice and non-locality provide the gupraluminal correlations experimentally demonstrated in EPR experiments without allowing a upraluminal transmission of information. As is true for any quantum mechanlcal theory, ours stands because of the outcome of Aspect's and gimilar experiments, and would have to fall if these are rejected. We claim to have arrived at an objective quantum mechanics with all the needed properties." 


\section{REFERENCES}

1. Wheeler, J.A. 1983. Law Without Law.In J.A.Wheeler \& W.H.Zurek, eds., Quantum Theory and Measurement, Princeton University Press: 182-213.

2. Noyes, H.P., C.Gefwert \& M.J. Manthey. 1985. In Proc., Symposium on the Foundotions of Modern Phyries, Joensuu, Finland 16-20 June, (in press). See also H.P.Noyes, C.Gefwert and M.J.Manthey, "Toward a Constructive Physics", SLAC-PUB-3116 (rev. September 1983), and references therein.

3. Gefwert, C. 1983-84. A Participator: the Metaphysical Subject, SLAC-PUB3277 (December, 1983) and The Proposition-ng-Rules Idea, SLAC-PLB3303, (March 1944), to be submitted to Synthese. On The Logical Form of Primitive Recursive Funetions, SLAC-PUB-3334 \{May 1981\} and On The Logical Form of Mathematical Language, SLAC-PUB-3344 (May 1984), to be submitted to the Journal of Philosophical Logic.

4. Bastin, T. 1966. Studia Phiosophica Ganderuia, 4: 77.

5. Noyes, H.P.,G. Pastrana \& J.V.Lindegay. 1986. “A Marifestly Covariant Linear S-Matrix Dynamics Exhibiting Exact Unitarily and 'Crossing Symmetry" (in preparation). H.P.Noyes \& J.V.Lindesay, A ustralian J. Phys., 38: 601 (1983). J.V.Lindesay, A.J.Markevich, H.P.Noyes \& G.Pastrani, “A Self-Consistent, Poincare Invariant, and Unitary Three-Particle Scattering Theory", SLAC-PUB-3661, May, 1985 and Phys, Reu.D (in press).

6. McGoveran, D. 1985. $7^{\text {th }}$ Annual Internztional Meeting of the Alternalive Natural Philosophy Association, King's College, Cambridge, (unpublished) and private communication.

7. Parker-Rhodes,A.F. 1981. The Theory of Indistinguishables, Synthese Ljbrary, 150, Reidel, Dordrecht: 182-185. 
Table 1

The combinatorial hierarchy

hietarchy

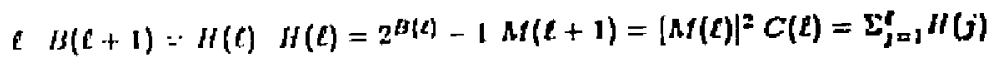

$$
\text { lavel }
$$

$\begin{array}{cc}(0) & - \\ 1 & 2 \\ 2 & 3 \\ 3 & 7 \\ 4 & 227\end{array}$

2
3
7
127
$2^{127}-1$

(2)

13

I6. 10

$256 \quad 337$

$202 \quad(256)=2127-1 \quad 2^{137}-1+137$

Level 5 canrot be consiructed because $M(1)<I(1)$ 


\section{Table 2}

The SU3 octet for "I,U,V spin"

STRING: $\begin{array}{cccc}\left(b_{1} b_{2} b_{3} b_{4}\right) & 2 I_{z} & 2 U_{z} & \left.2 V_{x}=2\left(I_{z}+U_{z}\right)\right] \\ 1110 & +1 & +1 & +2 \\ 0010 & -1 & +2 & +1 \\ 1100 & +2 & -1 & +1 \\ 1111 & 0 & 0 & 0 \\ 0000 & 0 & 0 & 0 \\ 0011 & -2 & +1 & -1 \\ 1101 & +1 & -2 & -1 \\ 0001 & -1 & -1 & -2\end{array}$

$2 I_{2}=b_{1}+b_{2}-b_{3}-b_{4}$

$2 U_{2}=-2 b_{1}+b_{2}+2 b_{3}-b_{4}$

$2 V_{x}=-b_{t}+2 b_{2}+b_{3}-2 b_{1}$ 


\section{FIGURE CAPTIONS}

1. The relation between knowledge of meaning and knowledge of fact, theory and measurement for a research program in physics.

2. Program Universe.

3. How events happen in Program Universe.

4. The quaniwin numbers for a string $\left(b_{1}, b_{2}, b_{3}, b_{4}\right)$ defined by $q_{1}=b_{1}-b_{2}+$ $b_{3}-b_{1}$ and $q_{2}=b_{1}+b_{2}-b_{2}-b_{4}$ plotted on a square mesh and $2 q_{1}, 2 q_{2}, q_{1}+q_{2}$ platted on a hexagonal mesh.

5. The connection between the address atrings in tick-separated events resulting from an initial uncertainty in velocity mensurement: If $k, k^{\prime}$ represenc two values of $k$ allowed by the velocity uncertainty $\Delta \beta$, and $\Delta k$ the corresponding integral uncertainty, the correlated probability of having both, normalized to unity when they are the same is $f\left(k, k^{\prime}\right)=\frac{2 \Delta k=\left(k^{\prime}-k\right)}{2 \Delta k \pm\left(k^{\prime}-k\right)}$, where the positive sign corresponds to $k^{\prime}>k$. The correlated probability of finding two values $k_{T}, k_{T}^{\prime}$ after $T$ licks in an event with the same labels and same normalization is $\frac{f\left(k_{T}, k_{j}^{*}\right)}{f\left(k, k^{\prime}\right)}$. This is 1 if $k^{\prime}=k$ and $k_{T}^{\prime}=k_{T}$. But outside of this specific requirement, we can see that this ratio. written as

$$
\frac{1 \pm \frac{2\left(\Delta k-\Delta k_{T}\right)}{\left(k^{2}-k\right)}+\frac{4 \Delta k \Delta k_{T}}{(k-k)^{2}}}{1 \mp \frac{2\left(\Delta k-\Delta k_{T}\right)}{\left(k^{\prime}-k\right)}+\frac{\left(\Delta k \Delta k_{T}\right.}{(k-k)^{2}}}
$$

gocs to $0^{*}$ in the large number or sharp resolution limits, thus correlating the limits to an ordering depending on the sign of the velocity. 


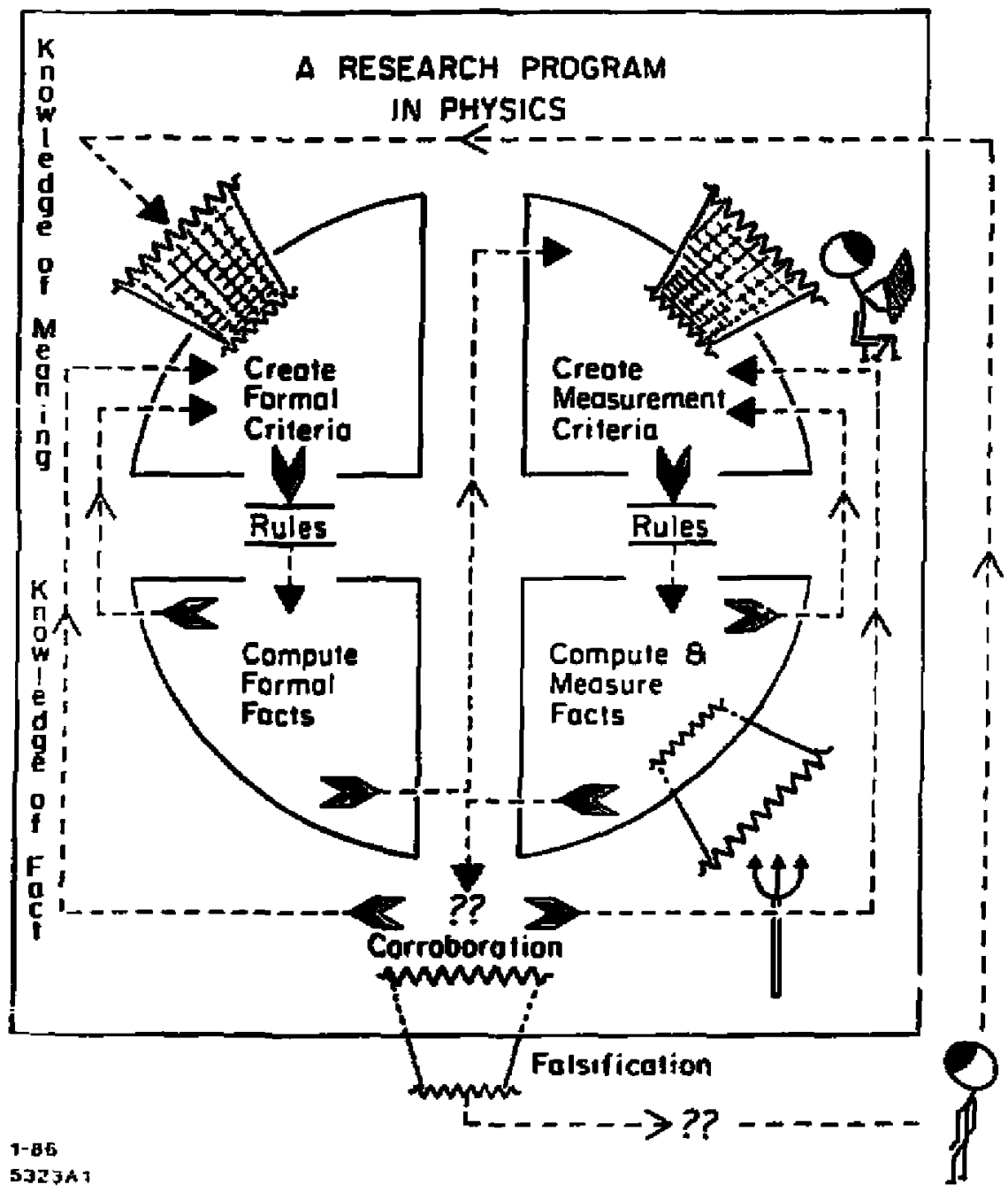

Fig. 1 


\section{PROGRAM UNIVERSE}

NO. STRIXGS $=S U \quad R \Rightarrow 0,1$ (FLIP BIT)

LENGTH $=\kappa_{\mathrm{l}} ; \quad$ PICK $:=$ SOME $U[i] \quad p=\mathrm{J} / \mathrm{SU}$

ELEMENT U[i] TICK $\mathbf{U}:=\mathbf{U} \| \mathbf{R}$

$i \in 1,2 \ldots, N \quad \bar{S}=I_{N} \oplus S$

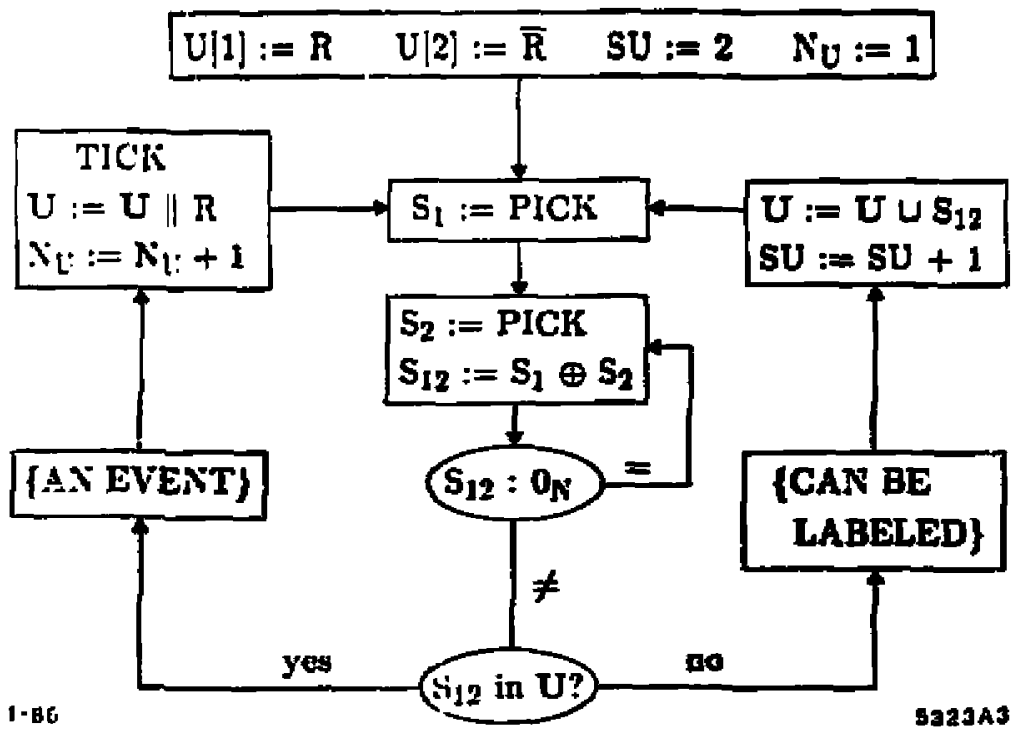

Fig. 2 


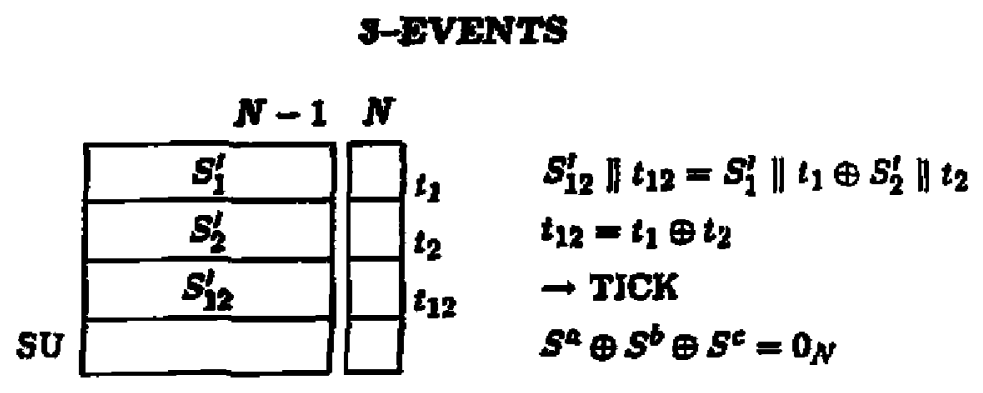

A-DVENTS

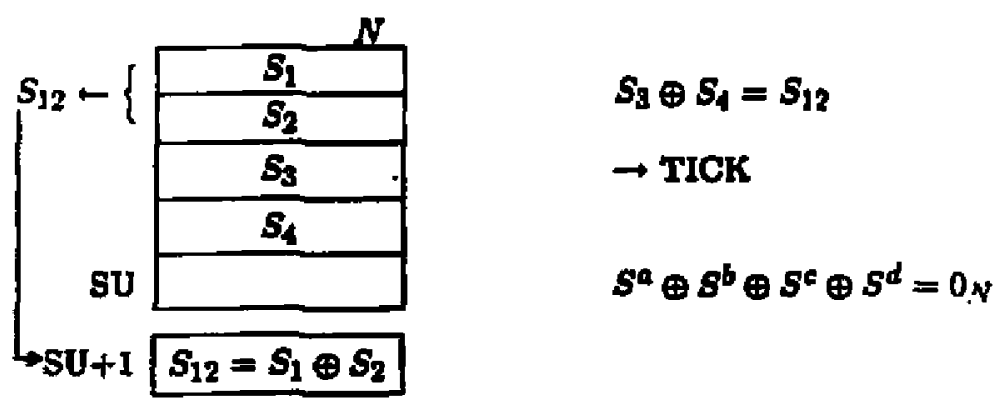

EACH TICK "RECORDS" A UNIQUE EVENT "SOMEWHERE" IN THE UNTVERSE

Fig. 3 


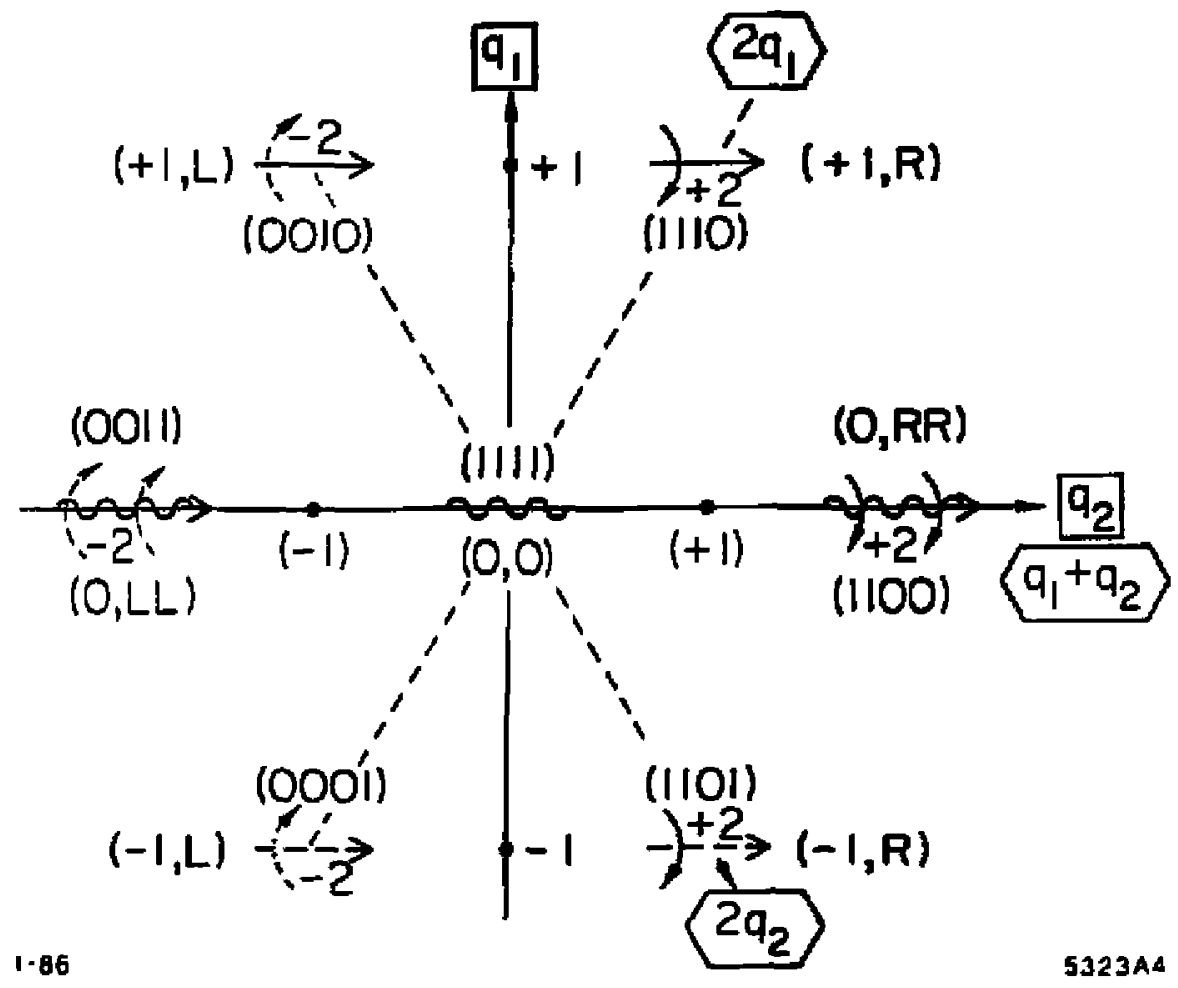

Fig. 4 


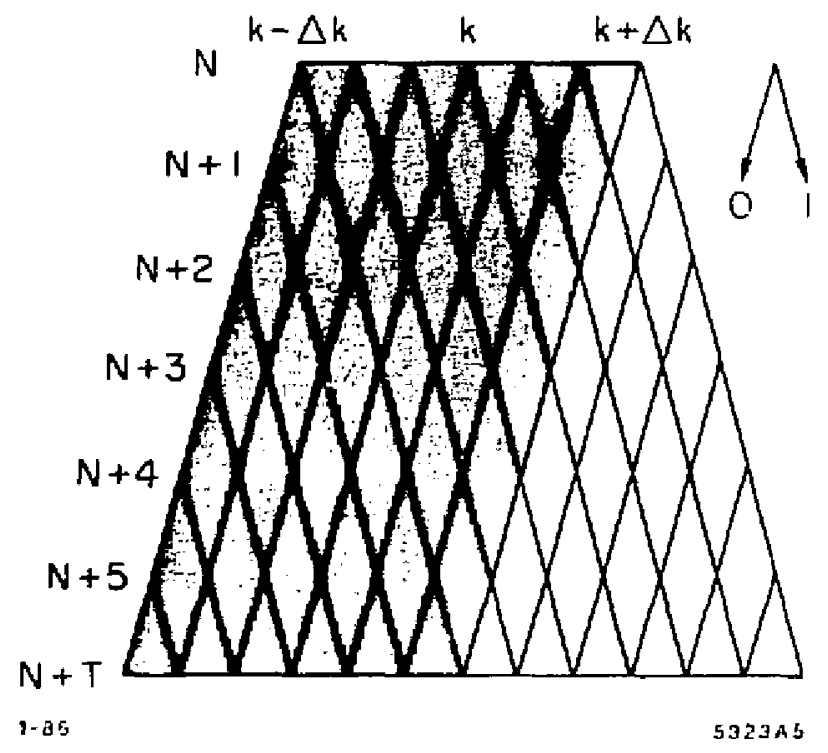

Fig. 5 